Pacific Journal of Mathematic 


\title{
LOCALITY OF THE NUMBER OF PARTICLES OPERATOR
}

\author{
M. ANN PIECH
}

\begin{abstract}
We view the number of particles operator $N$ as the infinitesimal generator of the Ornstein-Uhlenbeck semigroup in an abstract Wiener setting. It is shown that if two functions $f, g$ in the domain of $N$ agree a.e. on an open set $\mathcal{O}$, then $N f=N g$ on $O$. The restriction of $N$ to a large core acts as an infinite dimensional partial differential operator $L$, and it is shown that $L$ may be defined locally in an $L_{100}^{2}$ setting.
\end{abstract}

One of the mathematical concepts which has been the subject of considerable recent interest in constructive quantum field theory is the identification of the Bose Fock space $\mathscr{F}$ with a space $\mathscr{L}$ of $L^{2}$ functions over some Gaussian measure space $(\mathscr{Q}, d \mu)$. When $\mathscr{F}=$ $\sum_{n=0}^{\infty} \bigotimes_{n}^{s} \mathscr{H}$, the sum of the $n$-fold Hilbert space symmetric tensor product of the complexification $\mathscr{H}$ of a real separable Hilbert space $H$, possible choices of $(\mathscr{Q}, d \mu)$ include any measure space on which the isonormal distribution over $H$ may be realized. This identification is nicely described by Nelson [3]. The isometric isomorphism of $\mathscr{F}$ with $\mathscr{L}$ preserves the canonical direct sum decomposition of $\mathscr{F}$; that is, we have a corresponding decomposition $\mathscr{L}=\sum_{n=0}^{\infty} \mathscr{L}_{n}$. $\mathscr{L}_{n}$ has a natural interpretation as the $L^{2}$ space spanned by the Hermite functions on $\mathscr{Q}$ of rank $\leqq n[3,8]$.

One way of realizing the isonormal distribution on $H$ is to complete $H$ with respect to a weaker norm (a "measurable" norm in the sense of Gross [2]) obtaining a Banach space $B$ in which $H$ is densely embedded. The pair $(H, B)$ is known as an abstract Wiener pair, and $\mu$ is taken to be the Wiener measure $p_{1}$ on the Borel sets of $B$, generated by the canonical Gauss cylinder set measure on $H$ [2]. Under the identification of $\mathscr{F}$ and $L^{2}\left(p_{1}\right)$, we further identify the number of particles operator on $\mathscr{F}$ (i.e. the second quantization of the identity operator on $H$ ) with the infinitesimal generator $N$ of the Ornstein-Uhlenbeck velocity semigroup $\left\{e^{-t N}\right\}$ for the Brownian motion on $B[3,4]$. For $H$ finite dimensional $N f(x)=-\Delta f(x)+x \cdot \operatorname{grad} f(x)$ on smooth $f$. However, $\mathscr{F}$ is usually constructed over an infinite dimensional $H$, and the expression of $N$ as a differential operator must be suitably reinterpreted.

As a differential operator, $N$ only incorporates derivatives in the directions of vectors of $H$. We define the $H$-derivative $D g(x)$ of a function $g$ defined on a neighborhood of $x$ in $B$ and taking values in a Banach space $W$ as follows. Let $\hat{g}(h)=g(x+h)$ for all $h$ in $H$ 
such that $x+h$ is in the domain of $g$. Then $\hat{g}$ maps a neighborhood of the origin of $H$ into $W . \quad D g(x) \equiv \hat{g}^{\prime}(0)$, the Fréchet derivative of $\hat{g}$ at 0 . When $g$ is real valued, $D g(x)$ is an element of $H^{*}$ which is customarily identified via the Riesz representation with an element of $H . \quad D^{2} g(x)$ is defined by iteration, and will be identified with an element of $\mathscr{L}(H, H)$. Since $B^{*}$ is dense in $H^{*} \approx H$, we can always find orthornormal bases $\left\{e_{i}\right\}$ for $H$ consisting of elements of $B^{*}$.

In [4] it is shown that the set

$$
\begin{aligned}
\mathscr{C}= & \left\{\text { real valued } f \in L^{2}\left(p_{1}\right) \text { such that }|D f(x)|_{H}\right. \text { exists } \\
& \text { a.e. and is in } L^{2}\left(p_{1}\right) \text { and also } D^{2} f(x) \text { exists a.e. } \\
& \text { and is a Hilbert-Schmidt operator on } H \text { with } \\
& \left.\left|D^{2} f(x)\right|_{\mathscr{C}-\mathscr{S}} \in L^{2}\left(p_{1}\right)\right\}
\end{aligned}
$$

is a core for $N$. The action of $N$ on an $f$ in $\mathscr{C}$ is as follows. If $\left\{e_{i}\right\}$ is any orthornormal basis in $H$ with $e_{i} \in B^{*}$, and $P_{i}$ is the orthogonal projection of $H$ onto $\left\{e_{1}, \cdots, e_{i}\right\}$, then

$$
\left\{\left\langle x, P_{i} D f(x)\right\rangle-\operatorname{trace}\left(P_{i} D^{2} f(x)\right)\right\}_{i=1,2, \cdots}
$$

is a Cauchy sequence in $L^{2}\left(p_{1}\right) . N f$ is the limit of this sequence, and is independent of the choice of $\left\{e_{i}\right\}$.

Other smaller cores for $N$ are well-known. They generally consist of smooth polynomial cylinder functions. For the purposes of this note, however, $\mathscr{C}$ possesses a property that polynomial cores fail to have. Namely, the elements of $\mathscr{C}$ suffice to generate partitions of unity on $B[1,6]$. That is, given any two concentric balls $b_{1} \subsetneq b_{2}$ in $B$, we can find $\varphi \in \mathscr{C}$ such that $0 \leqq \varphi(x) \leqq 1, \varphi(x)=1$ on $b_{1}$ and $\varphi(x)=0$ on $B-b_{2}$. Moreover, $\varphi(x),|D \varphi(x)|_{H}$ and $\left|D^{2} \varphi(x)\right|_{\not C-S}$ can be assumed continuous and bounded on $B$. We call such a $\varphi$ a partition function for $b_{1}, b_{2}$. We point out that if $H$-differentiability were replaced with the usual Fréchet differentiability on $B$, it would not always be possible to find a nontrivial $C^{1}$ function $\varphi$ vanishing off $b_{2}$.

Locality of $N$ can be stated in several ways. If two functions $f, g$ in the domain of $N$ have disjoint supports, then $N f$ and $N g$ have disjoint supports. Or, a stronger statement, that if $f$ and $g$ coincide a.e. on an open set, then $N f=N g$ a.e. on that set. Or, equivalently,

Proposition 1. If $f$ is in the domain of $N$ and if $f$ vanishes a.e. on an open subset $\mathcal{O}$ of $B$, then $N f$ vanishes a.e. on $\mathcal{O}$.

Proof. Since $\mathscr{C}$ is a core for $f$, we can find $f_{n} \in \mathscr{C}$ with $f_{n} \rightarrow f\left(L^{2}\right)$ and $N f_{n} \rightarrow N f\left(L^{2}\right)$. Fix $y \in \mathcal{O}$, and choose two open balls 
$b_{1}, b_{2}$ centered at $y$, with $b_{1} \subset b_{2}$ and $\overline{b_{2}} \subset \mathcal{O}$. Choose $\varphi_{y} \in \mathscr{C}$ with $0 \leqq$ $\varphi_{y}(x) \leqq 1, \varphi_{y}(x)=0$ on $b_{1}, \varphi_{y}(x)=1$ on $B-b_{2}$ and with $\varphi_{y}(x),\left|D \varphi_{y}(x)\right|_{H}$ and $\left|D^{2} \varphi_{y}(x)\right|_{x-S}$ all continuous and bounded on $B$. Since $\partial b_{2}$ has $p_{1}$ measure zero, we may without loss of generality assume each $f_{n}$ vanishes on $b_{2}$. Now $\varphi_{y} f_{n} \rightarrow \varphi_{y} f=f$ in $L^{2}$. Also $\varphi_{y} f_{n} \in \mathscr{C}$, and

$$
\begin{aligned}
N \varphi_{y} f_{n}= & \lim _{i} \varphi_{y}(x)\left\{\left\langle x, P_{\imath} D f_{n}(x)\right\rangle-\operatorname{trace}\left(P_{i} D^{2} f_{n}(x)\right)\right\} \\
& +\lim _{i} f_{n}(x)\left\{\left\langle x, P_{i} D \varphi_{y}(x)\right\rangle-\operatorname{trace}\left(P_{i} D^{2} \varphi_{y}(x)\right)\right\} \\
& -2 \lim _{i} \operatorname{trace} P_{\imath}\left(D \varphi_{y}(x) \otimes D f_{n}(x)\right) .
\end{aligned}
$$

Dominated convergence ensures that the first limit exists, and the choice of support for $f_{n}$ ensures that the subsequent terms are zero a.e. Hence $N \varphi_{y} f_{n}=\varphi_{y} \cdot N f_{n}$, and so $N \varphi_{y} f_{n} \rightarrow \varphi_{y} \cdot N f$ in $L^{2}$. Since $N$ is closed, $\varphi_{y} \cdot N f=N f$ follows. Thus $N f$ vanishes a.e. on $b_{1}$. Since $B$ is separable, it follows that $N f$ vanishes a.e. on $O$.

It is expected that $N$ should serve as the model for the LaplaceBeltrami operator on manifolds modelled on $B$. We will now show that we can easily locally define an operator $L$ which extends the restriction of $N$ to $\mathscr{C}$. For any open subset $\mathcal{O}$ of $B$, we define

$$
\begin{aligned}
\mathscr{C}_{\odot}= & \text { real valued } f \text { defined on } \mathcal{O} \text {, with }|D f(x)|_{H} \\
& \text { and }\left|D^{2} f(x)\right| \mathscr{C}-\mathscr{S} \text { existing a.e. on } \mathcal{O} \text {, such that } \\
& f,|D f|_{H} \text { and }\left|D^{2} f\right|_{\mathscr{C}-S} \text { are locally in } L^{2}\left(p_{1}\right) \text { on } \\
& \mathcal{O}\}
\end{aligned}
$$

Then we may define $L$ on $\mathscr{C}_{0}$ by

Proposition 2. Given $f$ in $\mathscr{C}_{Q}$, let $\left\{\mathscr{O}_{n}\right\}$ by any countable cover of $\mathcal{O}$ by open balls such that for each $\mathcal{O}_{n}$ there is a concentric $\mathcal{O}_{n}^{\prime}$ with $\mathscr{O}_{n} \subsetneq \mathcal{O}_{n}^{\prime} \subset \mathcal{O}$ and such that $f,|D f|_{H}$ and $\left|D^{2} f\right|_{\mathscr{C}-s}$ are in $L^{2}$ on each $\mathscr{O}_{n}^{\prime}$. Let $\varphi_{n}$ be a partition funcion for $\left\{\mathscr{O}_{n}, \mathscr{O}_{n}^{\prime}\right\}$. Extend $\varphi_{n} f$ to be zero outside $\mathcal{C}$. Then $\varphi_{n} f \in \mathscr{C}$, and we define $L f=N \varphi_{n} f$ on $\mathcal{O}_{n}$. Then $L f$ is well defined, is locally in $L^{2}\left(p_{1}\right)$ on $\mathcal{O}$, and is independent of the choice of $\mathcal{O}_{n}$ and $\varphi_{n}$.

Proof. If $x$ belongs to two members of the covering, say to $\mathcal{O}_{n}$ and $\mathscr{O}_{m}$, then $\varphi_{n} f$ and $\varphi_{m} f$ agree on $\mathscr{O}_{n} \cap \mathcal{O}_{m}$ and $L f$ is welldefined by Proposition 1 . Hence since $B$ is separable, $L f$ is independent of the choice of $\mathcal{O}_{n}$ and $\varphi_{n}$.

In Reference [4] it is shown that for $f \in \mathscr{C}$,

$$
|N f|_{L^{2}\left(p_{1}\right)}^{2} \leqq\left.\left.|| D f\right|_{H I}\right|_{L^{2}\left(p_{1}\right)} ^{2}+\left.\left.|| D^{2} f\right|_{\mathscr{C}-s}\right|_{L^{2}\left(p_{1}\right)} ^{2} \cdot
$$

Thus for for $f$ in $\mathscr{C}_{0}$, it follows that $L f$ is square integrable on $\mathscr{O}_{n}$. 
REMARK. A popular choice of $(\mathscr{Q}, d \mu)$ is the underlying probability space of the realization on $\mathscr{S}^{\prime}\left(\boldsymbol{R}^{d}\right)$ of a Gaussian process over Schwartz space $\mathscr{S}\left(\boldsymbol{R}^{d}\right)$. That is, $\mathscr{Q}=\mathscr{S}^{\prime}$ and $d \mu$ is a Gaussian Borel measure on $\mathscr{S}^{\prime}$. Such measures $d \mu$ have as supporting sets Hilbert spaces $B \subset \mathscr{S}^{\prime}$, such that there is an $H \subset B$ with $(H, B)$ an abstract Wiener pair. $\left.d \mu\right|_{B}=p_{1}$, the Wiener measure for $(H, B)[7$, 5]. Our Proposition 1 then may be applied in $L^{2}\left(B, p_{1}\right)$.

\section{REFERENCES}

1. V. Goodman, Quasi-differentiable functions on Banach spaces, Proc. Amer. Math. Soc., 30 (1971), 367-370.

2. L. Gross, Abstract Wiener spaces, in Proceedings of the Fifth Berkeley Symposium and Probability, 1963, 31-42.

3. E. Nelson, Probability theory and Euclidean field theory, in "Lecture Notes in Physics" v 25, Springer-Verlag, Berlin, 1973.

4. M. A. Piech, The Ornstein-Uhlenbeck semigroup in an infinite dimensional $L^{2}$ setting, J. Functional Analysis, 18 (1975), 271-285.

5. - Support properties of Gaussian processes over Schwartz space, Proc. Amer. Math. Soc., 53 (1975).

6. — Smooth functions on Banach spaces, J. Math. Anal. and Appl., to appear. 7. M. Reed and L. Rosen, Support properties of the free measure for Boson fields, Comm. Math. Physics, 36 (1974), 123-132.

8. I. E. Segal, .Tensor algebras over Hilbert spaces, Trans. Amer. Math. Soc., 81 (1956), 106-134.

Received July 11, 1975. Research supported by NSF grant PO-28934.

State University of New York at Buffalo 


\section{PACIFIC JOURNAL OF MATHEMATICS}

\section{EDITORS}

RICHARD ARENS (Managing Editor) University of California

Los Angeles, California 90024

\section{R. A. BEAUMONT}

University of Washington Seattle, Washington 98105
J. DugundjI Department of Mathematics University of Southern Californı Los Angeles, California 90007

D. Gilbarg AND J. Milgram Stanford University Stanford, California 94305

\section{ASSOCIATE EDITORS}

E. F. BECKENBACH

B. H. NeUMANN

F. WOLF

K. YosHidA

\section{SUPPORTING INSTITUTIONS}

UNIVERSITY OF BRITISH COLUMBIA CALIFORNIA INSTITUTE OF TECHNOLOGY UNIVERSITY OF CALIFORNIA MONTANA STATE UNIVERSITY UNIVERSITY OF NEVADA NEW MEXICO STATE UNIVERSITY OREGON STATE UNIVERSITY UNIVERSITY OF OREGON OSAKA UNIVERSITY

\author{
UNIVERSITY OF SOUTHERN CALIFORNIA \\ STANFORD UNIVERSITY \\ UNIVERSITY OF TOKYO \\ UNIVERSITY OF UTAH \\ WASHINGTON STATE UNIVERSITY \\ UNIVERSITY OF WASHINGTON \\ AMERICAN MATHEMATICAL SOCIETY \\ NAVAL WEAPONS CENTER
}




\section{Pacific Journal of Mathematics}

\section{Vol. 61, No. $1 \quad$ November, 1975}

Jiří Adámek, V. Koubek and Věra Trnková, Sums of Boolean spaces represent every

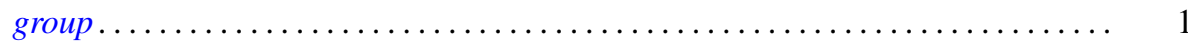

Richard Neal Ball, Full convex l-subgroups and the existence of $a^{*}$-closures of

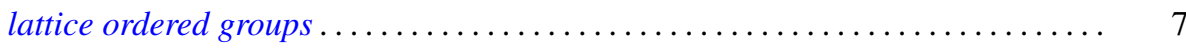

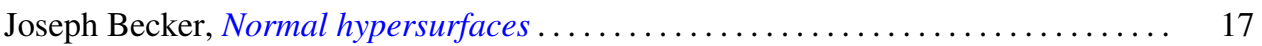

Gerald A. Beer, Starshaped sets and the Hausdorff metric . . . . . . . . . . . . . 21

Dennis Dale Berkey and Alan Cecil Lazer, Linear differential systems with

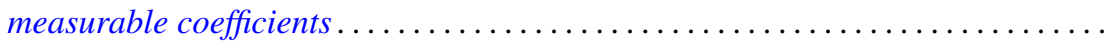

Harald Boehme, Glättungen von Abbildungen 3-dimensionaler

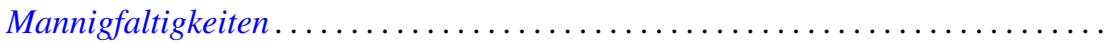

Stephen LaVern Campbell, Linear operators for which $T^{*} T$ and $T+T^{*}$

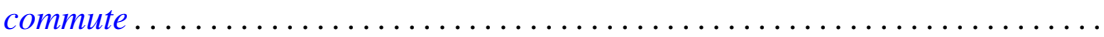

H. P. Dikshit and Arun Kumar, Absolute summability of Fourier series with

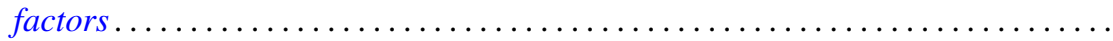

Andrew George Earnest and John Sollion Hsia, Spinor norms of local integral

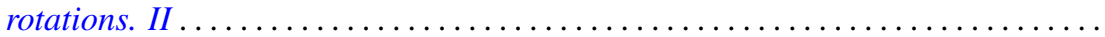

Erik Maurice Ellentuck, Semigroups, Horn sentences and isolic structures .........

Ingrid Fotino, Generalized convolution ring of arithmetic functions . . . . . . . . . . .

Michael Randy Gabel, Lower bounds on the stable range of polynomial rings .......

Fergus John Gaines, Kato-Taussky-Wielandt commutator relations and

characteristic curves

Theodore William Gamelin, The polynomial hulls of certain subsets of $C^{2}$

R. J. Gazik and Darrell Conley Kent, Coarse uniform convergence spaces. . .

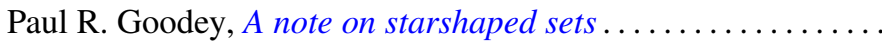

Eloise A. Hamann, On power-invariance

M. Jayachandran and M. Rajagopalan, Scattered compactification for $N \cup\{P\}$. . .

V. Karunakaran, Certain classes of regular univalent functions .

John Cronan Kieffer, A ratio limit theorem for a strongly subadditive set function in a locally compact amenable group .................

Siu Kwong Lo and Harald G. Niederreiter, Banach-Buck measure, density, and uniform distribution in rings of algebraic integers ........

Harold W. Martin, Contractibility of topological spaces onto metric spaces ....

Harold W. Martin, Local connectedness in developable spaces .

A. Meir and John W. Moon, Relations between packing and covering numbers of a tree.

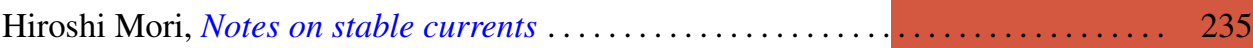

Donald J. Newman and I. J. Schoenberg, Splines and the logarithmic function . . . . 241

M. Ann Piech, Locality of the number of particles operator....

Fred Richman, The constructive theory of $K T$-modules .......

Gerard Sierksma, Carathéodory and Helly-numbers of

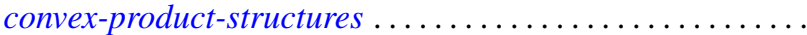

Raymond Earl Smithson, Subcontinuity for multifunctions .... . . 